\title{
A new approach to the anthropocentric design of human- robot collaborative environments
}

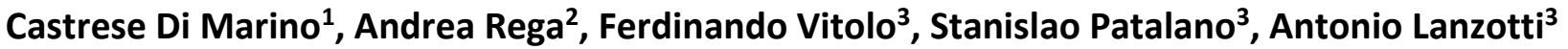 \\ ${ }^{1}$ Department of Management, Information and Production Engineering, University of Bergamo, Bergamo, Italy \\ 2 Department of Neurosciences, Reproductive and Odontostomatological Sciences, University of Naples 'Federico II', Naples, Italy \\ ${ }^{3}$ Fraunhofer J-Lab IDEAS, Deptartment of Industrial Engineering, University of Naples 'Federico II', Naples, Italy
}

\begin{abstract}
This paper deals with collaborative robotics by highlighting the main issues linked to the interaction between humans and robots. $A$ critical study of the standards in force on human-robot interaction and the current principles on workplace design for human-robot collaboration (HRC) are presented. The paper focuses on an anthropocentric paradigm in which the human becomes the core of the workplace in combination with the robot, and it presents a basis for designing workplaces through two key concepts: (i) the introduction of human and robot spaces as elementary spaces and (ii) the dynamic variations of the elementary spaces in shape, size and position. According to this paradigm, the limitations of a safety-based approach, introduced by the standards, are overcome by positioning the human and the robot inside the workplace and managing their interaction through the elementary spaces. The introduced concepts, in combination with the safety prescriptions, have been organised by means of a multi-level graph for driving the HRC design phase. The collaborative workplace is separated into sublevels. The main elements of a collaborative workplace are identified and their relationships presented by means of digraphs.
\end{abstract}

\section{Section: RESEARCH PAPER}

Keywords: Anthropocentric approach; human-robot collaboration; collaborative environment; graph theory; digraph

Citation: Castrese Di Marino, Andrea Rega, Ferdinando Vitolo, Stanislao Patalano, Antonio Lanzotti, A new approach for an anthropocentric design of human-robot collaborative environments, Acta IMEKO, vol. 9, no. 4, article 11, December 2020, identifier: IMEKO-ACTA-09 (2020)-04-11

Section Editor: Leopoldo Angrisani, University of Naples Federico II, Italy

Received October 31, 2019; In final form November 19, 2020; Published December 2020

Copyright: This is an open-access article distributed under the terms of the Creative Commons Attribution 3.0 License, which permits unrestricted use, distribution, and reproduction in any medium, provided the original author and source are credited.

Corresponding author: Castrese Di Marino, e-mail: castrese.dimarino@unibg.it

\section{INTRODUCTION}

Industry 4.0 pillars and technological improvements are pushing manufacturing towards hyper-automated production lines [1]-[3]. However, many production applications cannot be implemented without a human presence; many assembly processes need to be performed with human involvement [4], [5]. These applications, usually based on multi-stage processes [6], are often characterised by a sequence of human- and robot-led stages.

Indeed, many researchers assume humans to be the central actors in manufacturing plants [6]-[8] because they cannot be easily replaced by advanced technologies [6].

According to several researchers [9], [10], novel workplaces can achieve higher productive levels by combining (i) human capabilities (intelligence, flexibility and adaptability) and (ii) robot characteristics (strength, endurance and accuracy). Moreover, the synergic collaboration of humans and robots leads to new opportunities in ergonomics, such as a reduction in bad postures [11] and an increasing perception of well-being during work activities [12]. Therefore, a synergic combination of human and robotic skills should be considered as the key approach to improving manufacturing plants.

Currently, three kinds of workplace are used in manufacturing plants: (a) manual workplaces, where an operator performs all the tasks alone; (b) automatic workplaces, where robots autonomously perform all the tasks; and (c) collaborative workplaces, i.e. hybrid workplaces where humans and robots work together by performing common tasks, thus creating a human-robot collaboration (HRC).

Although workplaces (a) and (b) are common in manufacturing, workplace (c) is still not being implemented for a number of reasons, including safety, the difficulty of installation, suitability and reliability. Industry still lacks confidence in this kind of workplace.

However, hybrid workplace installations could have a large impact on (i) processes characterised by small-batch production 
(repetitive tasks, interaction with fixed objects), (ii) processes that are hard to automate (complex tasks, randomly positioned pieces, skill-based processes) and (iii) processes designed to relieve human effort (non-ergonomic and repetitive tasks, assembly support).

Of course, safety is the main issue that needs to be addressed. Within the current standards, it is clear how difficult it might be to assure safety in hybrid workplaces; to address all the standards in this context would be challenging and, sometimes, only a few indicators are provided [13]-[15].

A suggestion to overcome this difficulty is the adoption of engineering design methods that identify applications and requirements linked to the contents of the standards. One such method is to use axiomatic design [16] or graph theory, which will be presented in this paper, to find the functional requirements and the design parameters or the connections between the different elements of the workplace.

Moreover, according to the standards, a human presence is usually recognised as an intrusion (see safety-based monitored stops [13]) to be avoided whenever possible, and the designing of a workplace is safety-based and techno-centric, with a preference for static workspaces. All the effort goes into limiting the human presence in the workplace. Potential harm to humans is limited through hazard controls by means of protective stops, speed reduction and control system performance [14], [15]. All these precautions are mandatory requirements.

In hybrid workplaces, the usual approach consists of designing the workplace starting with the robot. The humanrobot interaction (collaborative operations) is then selected depending on the tasks, and the workplace spaces are defined (operative and collaborative space). Finally, the operator is added to help the robot perform all the tasks that it cannot carry out alone because of the lack of technology. This is the so-called 'techno-centric' design, i.e. all aspects are focused on the robot. Moreover, in the standards, there is no mention of a 'human workspace', a dedicated space where only the human presence is allowed.

Cognitive stress (mainly related to tracking the robot's movements) is another important topic in this field, and little research has been done in this direction [17]. Whenever a human is involved in these workplaces, ergonomic, cognitive and safety issues are the main areas of focus for the 'human-centred design'. The evaluation of the operator's stress levels [18] is a topic that needs to be studied to improve the quality of the work. Indeed, HRC represents an opportunity to improve not only the production but even the working conditions of the operators, who are liable to resign from heavy and repetitive jobs. Physical improvements need to be accompanied by mental improvements so that the operators can feel comfortable working with a robot. There is no trust or confidence in something they do not understand.

In the literature, many researchers have studied different approaches [19], [20] for designing human-robot workplaces based on (i) the different modalities of interaction and (ii) workspaces characterised by access properties. In [18] and [19], the authors define three dynamic areas with selective access (safe, warning and unsafe areas) and the way the robot system should react to the human presence. In [20], more details about levels of interaction are provided, and the static and dynamic space concepts are introduced. They move from a safety-based approach to one based on interaction assessment. A 'humancentred design' [21] could be adopted to overcome the current framework. Hence, a new prospective design based on an 'anthropocentric design' approach is proposed: humans and their interaction with the robot should be at the core of the workplace and design process.

Furthermore, with the large use of automated guided vehicles (AGV) and intelligent guided vehicles, standards for collaborative workplaces must not only address static robots. The use of AGVs is increasing. Generally, they are used for the internal and external transport of material, but they could also be employed to automate processes, such as in the reverse engineering of large objects [22].

Mobile robots (on rails or carried by autonomous systems) continuously move the reference frame and, therefore, workspaces are constantly changing. The definition of dynamic space is a very challenging topic [19], [23].

In this paper, the basis for an anthropocentric design is highlighted, focusing on two main topics: (i) the definition of elementary spaces for collaborative workplaces and (ii) the dynamic variations of elementary spaces in order to lead the designer towards the creation of a collaborative workplace and layout definition. Moreover, a multi-level graph-based approach for the design of an HRC workplace is presented for driving the HRC design phase.

In the rest of the paper, Section 2 introduces the standards and literature definitions, Section 3 presents the proposed spaces in HRC and Section 4 describes how the proposed approach influences collaborative operations. In Section 5, an approach using graph theory is presented, and, finally, the conclusions are set out in Section 6.

\section{BACKGROUND DEFINITIONS}

The composition of the workplace, provided by the ISO standards [13]-[15], is the following (Figure 1):

- 'Maximum space' is the space that can be swept by the whole robot system, made up of the robot's moving parts, the end effector and the workpiece.

- 'Safeguarded space' is the space limited by the safeguarding perimeter.

- 'Restricted space' is a part of the maximum space that is restricted by limiting devices, and it establishes a limit that cannot be exceeded.

- 'Operational space' is a part of the restricted space that can be used to perform all the actions commanded by the task programme.

- 'Collaborative workspace' is the space, within the operating space, where the whole robot system (robot arm, end effector and workpiece) and a human can

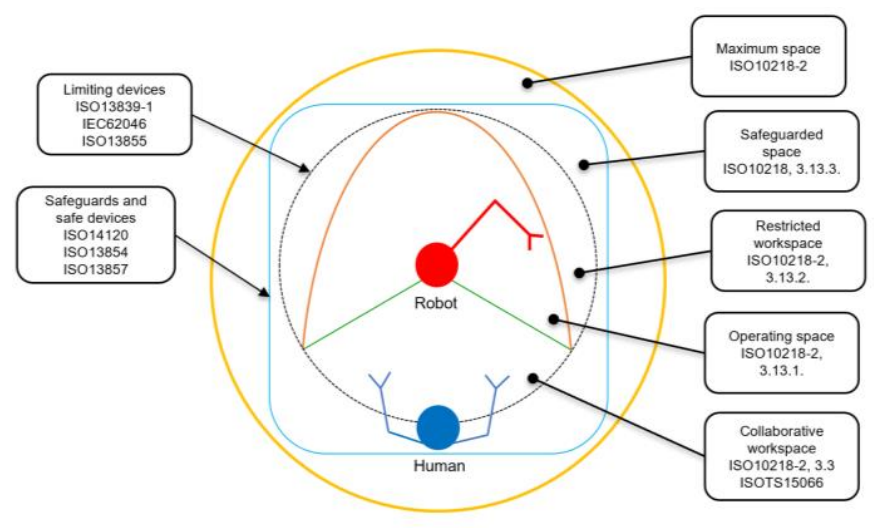

Figure 1. Collaborative workspaces according to ISO standards 


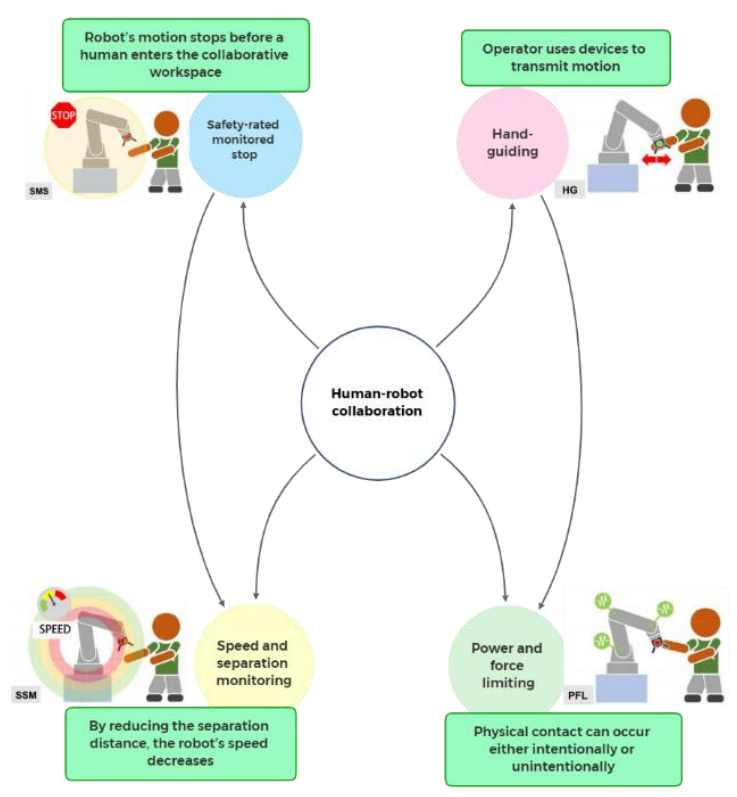

Figure 2. Collaborative operations within ISO/TS 15066 (also cited in [1])

concurrently perform tasks during production operations.

Furthermore, the collaborative operations [13] can be summarised as follow (Figure 2):

- $\quad$ Safety-rated monitored stop (SRMS): the robot ceases all movement before a human enters the collaborative space; after the human has exited the collaborative workspace, the robot can resume its movements.

- Hand-guiding (HG): motion commands are transmitted to the robot system either directly by a human or through a hand-operated device; the task is carried out by manually actuating guiding devices.

- Speed and separation monitoring (SSM): the robot system and the operator may move concurrently in the collaborative workspace maintaining the protective separation distance for risk reduction; if the separation distance falls below the protective separation distance, the robot system stops; if the protective separation distance decreases or increases, the speed of the robot system decreases or increases accordingly.

- Power and force limiting (PFL): physical contact between the robot system (including the end effector and workpiece) and the human can occur intentionally or unintentionally.

In this paper, human-robot interactions are classified based on two principles: workspace sharing and time sharing [18], [20]. They can be used to define a 'collaborative environment' in terms of a collaborative interaction between humans and robots:

- Workspace sharing: robots and humans sequentially perform their tasks sharing the same workspace at different times.

- Time sharing: robots and humans concurrently perform their tasks without sharing the workspace.

Time sharing and workspace sharing can only exist within the collaborative space.

Therefore, a collaborative environment can be enabled whenever a human and a robot concurrently perform their tasks in a common space (space and time sharing).

\section{SPACES IN HUMAN-ROBOT COLLABORATION}

Having defined the collaborative environment, in which the human and robot can work together, this proposal now introduces the two elementary spaces, each one dedicated to either the human or the robot, in order to provide a definite spatial collocation. Interactions can occur by introducing a composed space through the combination of the elementary spaces (Figure 3).

\subsection{Elementary spaces}

The elementary spaces, in which the human and robot can each work, are the following:

- 'Human space' (H) is a space dedicated to the human and includes all the equipment and the necessary space for humans to perform their tasks.

- 'Robot space' $(\mathrm{R})$ is a space dedicated to the robot system and includes all the space necessary for the robot to execute its movements and perform its tasks.

Such spaces have their own features and properties that will be explained below. The combination of the elementary spaces leads to the composed spaces.

\subsection{Composed spaces}

Composed spaces are made by the combination of the elementary spaces as follows (Figure 3):

- 'Collaborative space' (C) is the dynamic intersection of the elementary spaces (robot space and human space).

- 'Operational space' $(\mathrm{O})$ is the combination of the human space and robot space and represents the space strictly necessary to carry out all the operations, including the collaborative space.

- 'Restricted perimeter' $(\mathrm{P})$ is a perimeter around the workplace; a violation leads to a protective stop on all the operations.

- 'Safeguarded space' (S) is the space delimited by the safeguarded devices.

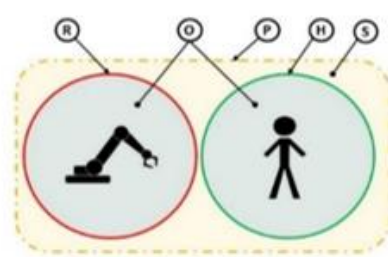

No interaction

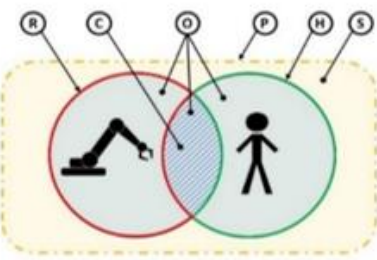

\section{Workspace}

sharing

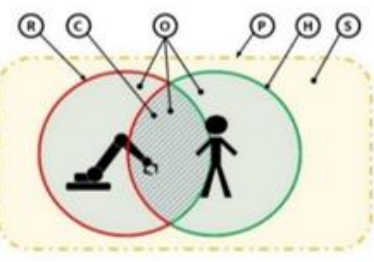

Time and

workspace

sharing

Figure 3. Significant spaces related to different human-robot interactions: $H$ Human space, R) Robot space, C) Collaborative space, P) Restricted perimeter, S) Safeguarded space, O) Operational space $(H+R+C)$. 
Table 1. Table of comparison

\begin{tabular}{lll}
\hline ISO Standards & Degree of interaction & Proposed approach \\
\hline SRMS & No interaction & Disjointed areas \\
\hline HG & Exploitation & No robot area \\
\hline SSM & Coexistence & $\begin{array}{l}\text { Intersection of areas - no } \\
\text { contact }\end{array}$ \\
\hline PFL & Collaboration & $\begin{array}{l}\text { Intersection of areas - } \\
\text { contact allowed }\end{array}$ \\
\hline
\end{tabular}

\subsection{Features of existing spaces}

By using sensors and cams, it is possible to dynamically change the elementary spaces in terms of shape, size and position:

- Position: related to the human and robot,

- Size: related to relative distance and speed,

- Shape: related to the direction of movement and task.

Therefore, the spaces are no longer static but can adapt dynamically to the task. The control system can modify them in accordance with the task sequence or in reaction to an unintended situation.

Space position is strictly related to the position of the human and robot during the task execution. Size is affected by the separation distance and the relative speed between the human and robot at any one time. Shape is affected by the relative direction of movement of the human and robot at any one time and in accordance with specific tasks.

As human and robot spaces can change during a task, the collaborative space can change as well.

Starting with the space definitions and their properties, the collaborative operations and rules for enabling the human-robot interaction can be defined.

\section{DESCRIPTION OF THE HUMAN-ROBOT INTERACTIONS BASED ON ELEMENTARY SPACES}

The current ISO standards for HRC describe only the behaviour of the robot according to different scenarios.

Indeed, the four collaborative operations (Section 2) represent a different degree of interaction between the human and robot (Table 1):

- SRMS: no interaction,

- HD: exploitation,

- SSM: coexistence,

- PFL: collaboration.

These collaborative operations can be used to better explain the different kinds of interaction in this proposed approach.

Therefore, according to the definitions of the elementary and composed spaces (sections 3.1 and 3.2), the collaborative operations in the standards can be reviewed and adapted to follow a task-oriented approach; the use of the proposed spaces is described below. It will then be made clear how the spacesbased approach can help to define the layout of the workplace leading to a collaborative environment.

\subsection{Safety-rated monitored stop}

In SRMS, there is no collaborative space or collaborative operations. The human can approach the workplace only when the robot is on stand-by mode. Any violation of the robot space generally causes a protective stop of the robot system; the human is seen as an intruder. Therefore, there is no real interaction between the human and robot and the safety is guaranteed by limiting the collaboration.

The human space and robot space are, ideally, overlapping and alternatively activated (workspace sharing). There is no time sharing, and a protective stop occurs to enable the human to enter the workplace by switching between the human and the robot space.

In this context, all the workplace features seem to belong to the robot. There is no space for the human operator. The layout definition is hindered by this lack of information since it is not clear to the designer where to place the human operator (even though SRMS may not consider the human presence).

According to the proposed approach, the human and robot spaces are simultaneously active but disjointed. Both human and robot have their own domain where they execute their tasks.

An intersection of spaces is not permitted while working (top of Figure 3), but the human presence is taken into account during the work and the layout design phase. This information helps the design process and allows for the optimisation of the provided spaces.

\subsection{Hand guiding}

In an HG operation, the robot can be seen as a tool in a human's hands. There is no robot space or human space because they cannot execute their tasks separately; in this case, the operational space is all the space required to carry out the task. However, hand guiding is not an actual collaborative operation since the human carries out all the tasks by handling the robot as a tool. There are no access properties for the working places, but there are switching procedures in order to guarantee human safety and enable the interaction.

Although such operations are characterised by both workspace sharing and time sharing, it seems incorrect to consider it to be a collaborative operation because it is characterised by an 'exploitation' of the robot's capabilities by the human (Table 1).

In this particular case, there is no actual robot space, even though this modality is rarely used alone but in combination with the automatic mode. The robot system switches to this modality in order to permit a human to take control of the robot.

Thus, this can be defined as the robot's domain during the collaboration and is characterised by two sub-domains: (i) the autonomous domain (outside the collaborative workspace) and (ii) the guided-movement domain, corresponding to the collaborative workspace (bottom of Figure 3). The designing of the spaces helps to define the layout because of the preliminary allocation of resources for the human and robot.

\subsection{Speed and separation monitoring}

In SSM, the human and robot can perform their tasks independently, sharing time and workspace. The separation distance between the human and robot needs to be monitored in real-time; a robot's speed is adjusted according to the safety rules and depending on the distance [13]. As for SRMS, direct and indirect contacts are not permitted; there are different safety distance levels, and a breach of a robot's nearest perimeter activates the robotic system's protective stop.

According to the current definition, the robotic system is the core of the workplace, where it can perform its own tasks, whereas the human operator can move around it, in an unspecified area, and without approaching within certain limits.

With the proposed approach, based on elementary spaces, the designer can provide a human and a robot with their own space 
where they can perform their tasks. During any interactions, such spaces can intersect and overlap, generating different configurations (the middle of Figure 3).

Using dynamic elementary spaces, it is possible to improve the interaction between humans and robots by adapting the position, size and shape.

\subsection{Power and force limiting}

In PFL, direct and indirect contacts are allowed. All necessary active and passive safety features are applied so that humans and robots can carry out their tasks, side by side, in a synergic and dynamic way. This represents a higher level of interaction and, possibly, the most dangerous.

Although this operation represents a complete HRC, it is not widely used due to the high levels of the operators' cognitive stress (i.e. not a predictable robot path) and the challenge of guaranteeing an appropriate level of safety (avoiding collisions and the balancing force).

Furthermore, the workplace design leans towards the robot system; the human operator can move around and work near the robot as a host.

In a complete HRC, the layout design process should consider humans and robots as peers. To improve the PFL, both humans and robots should be efficiently accommodated by not limiting their capabilities through a restrictive safety-based design. Therefore, a space dedicated to humans and another dedicated to robots should be clearly defined during the workplace design. Such spaces can be combined in order to better perform all the tasks.

The elementary spaces can intersect (collaborative space) and humans and robots can enter the collaborative space together to perform their tasks (bottom of Figure 3).

\subsection{Interactions: ISO vs elementary spaces}

A comparison between the current definition (ISO standards) and the proposed approach can be made. Table 1 summarises the innovations introduced by the elementary spaces.

According to the ISO standards, the collaborative operations represent a degree of interaction or a modality of operation (HG); thus, the designing process occurs around the robot. Instead, by adopting the elementary spaces, the designing process is carried out around the human-robot pair and the focus is switched towards the human.

Therefore, the basis for the design of the workplace layout becomes the identification of the human and robot spaces.

Table 2. Adjacency matrix for the HRC workplace at the second level.

\begin{tabular}{ccccccccccccc}
\hline- & PL & $\mathbf{C}$ & $\mathbf{E}$ & $\mathbf{I}$ & $\mathbf{E C}$ & $\mathbf{M}$ & $\mathbf{W}$ & $\mathbf{A}$ & $\mathbf{H I}$ & $\mathbf{R}$ & $\mathbf{P S}$ & $\mathbf{D}$ \\
\hline $\mathbf{P L}$ & 0 & 0 & 0 & 0 & 0 & 0 & 1 & 1 & 0 & 0 & 1 & 0 \\
\hline $\mathbf{C}$ & 0 & 0 & 0 & 0 & 0 & 0 & 1 & 1 & 1 & 0 & 1 & 1 \\
\hline $\mathbf{E}$ & 0 & 0 & 0 & 0 & 0 & 0 & 0 & 0 & 1 & 0 & 0 & 1 \\
\hline $\mathbf{I}$ & 0 & 0 & 0 & 0 & 0 & 0 & 1 & 0 & 1 & 1 & 0 & 1 \\
\hline EC & 0 & 0 & 0 & 0 & 0 & 0 & 1 & 0 & 1 & 0 & 0 & 0 \\
\hline $\mathbf{M}$ & 0 & 0 & 0 & 0 & 0 & 0 & 1 & 1 & 1 & 1 & 1 & 0 \\
\hline $\mathbf{W}$ & 0 & 0 & 0 & 0 & 0 & 0 & 0 & 1 & 0 & 0 & 1 & 1 \\
\hline $\mathbf{A}$ & 0 & 0 & 0 & 0 & 0 & 0 & 0 & 0 & 0 & 0 & 1 & 1 \\
\hline HI & 0 & 0 & 0 & 0 & 0 & 0 & 1 & 1 & 0 & 0 & 1 & 1 \\
\hline R & 0 & 0 & 0 & 0 & 0 & 0 & 1 & 1 & 1 & 0 & 1 & 1 \\
\hline PS & 0 & 0 & 0 & 0 & 0 & 0 & 0 & 0 & 0 & 0 & 0 & 0 \\
\hline
\end{tabular}

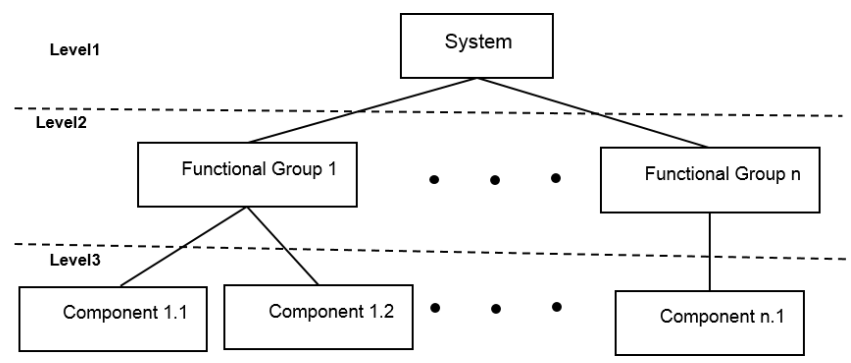

Figure 4. Multi-level approach

Furthermore, since the design process is performed around the human-robot pair, their interaction is placed at the core of the workplace, enabling a collaborative environment.

\section{WORKPLACE MULTI-LEVEL GRAPH-BASED MODELLING}

Workplace design involves a multitude of requirements, standard constraints, technological constraints and design parameters characterised by intertwined relations.

All these elements result in a dense network of relationships that is quite difficult to track and respect in the design phase. This aspect is more significant in the context of anthropocentric design due to the larger number of relations related to human space.

Dealing with such a multitude of relations requires a decomposition approach able to deepen and build the relations step by step.

Patalano et al. [24] proposed a multi-level approach based on graphs in the design of complex products using a digraph to manage and track the component relations.

Graph theory [25] provides many efficient tools to record and manage a large amount of information as well as to track their relationships. It provides an abstraction from the real model preserving all dependencies and relations between the elements involved. Graph theory is characterised by a set of nodes and edges $(G=\{N, E\})[26]$, and it uses an array or matrix for data organisation. The most significant matrix is the adjacency matrix that highlights the connection between the nodes; the elements of 1 or 0 mean the row element is either related to the column element or not. Direct graphs (digraphs) [27] were used in this paper. The nodes are connected by arrows that explain the relation between the two nearest nodes.

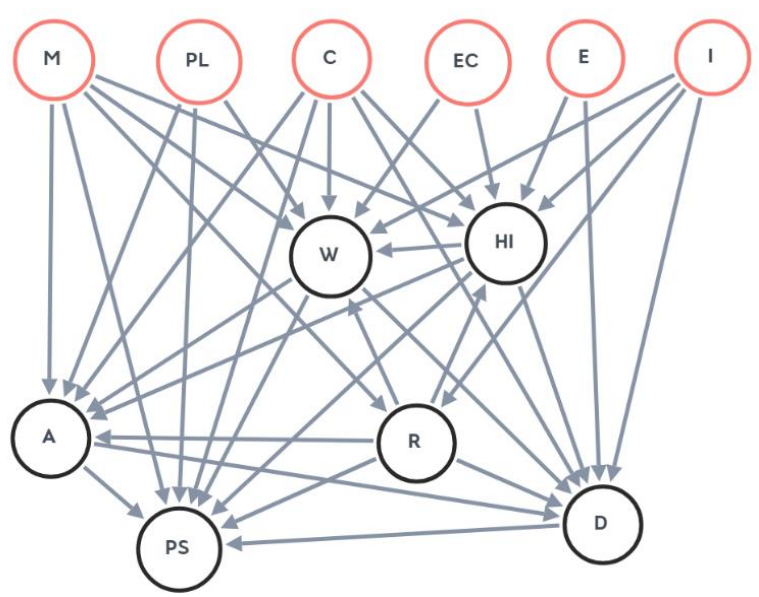

Figure 5. Second-level digraph representation for an HRC workplace; initial nodes are circled in red 


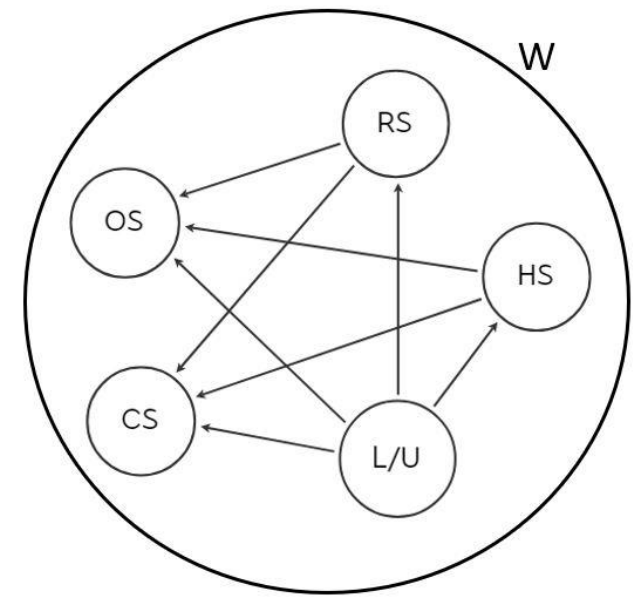

Figure 6. Third-level digraph representation for workspaces

Using a multi-level graph-based approach, it is possible to easily organise, exploit and manipulate the various elements of the workplace, helping to keep track of them. Indeed, graphs are used in several fields, such as networks of people, electronics, ergonomics and engineering, because of their versatility [28].

In the context of anthropocentric design, we have proposed three levels of detail (Figure 4) in which nodes are associated with different elements depending on their level of belonging, and directed edges always represent the relationships. In this abstract model of a workplace, the characterisation of the workplace comes from the combination of its basic elements, identified as nodes [25], where human intervention stands out from the beginning of the design process as a central element of the network, emphasising its importance. Thus, the human presence is no longer seen only as a constraint, for safety reasons, but, in conjunction with the robot, as the core of the workplace because of the interactions.

The whole collaborative workplace is assumed to be a firstlevel node. The second level is composed of functional elements defined by a logical decomposition. Such elements are called second-level nodes:

1. (PL) Physical Limit: physical limit of the cell

2. (C) Clearance: minimum distances required

3. (E) Ergonomics: ergonomic requirements

4. (I) Interaction: modality of interaction

5. (EC) Environmental Conditions: ventilation, welding sparks, etc.

6. (M) Material: material to be worked and tools

7. (W) Workspaces: areas inside the workplace

8. (A) Access: access routes and paths

9. (HI) Human Intervention: operator intervention

10. (R) Robot: robot characteristics

11. (PS) Perimeter Safeguarding: workplace border

12. (D) Devices: requirements of manual devices

Table 3. Adjacency matrix for workspaces (level-three detail)

\begin{tabular}{ccccccc} 
& & \multicolumn{5}{c}{ WORKSPACES (W) } \\
\cline { 3 - 7 } & & OS & CS & HS & RS & L/U \\
\cline { 2 - 7 } & OS & 0 & 0 & 0 & 0 & 0 \\
\cline { 2 - 7 } & CS & 0 & 0 & 0 & 0 & 0 \\
\cline { 2 - 7 } & HS & 1 & 1 & 0 & 0 & 0 \\
\cline { 2 - 7 } & RS & 1 & 1 & 0 & 0 & 0 \\
\cline { 2 - 7 } & L/U & 1 & 1 & 1 & 1 & 0
\end{tabular}

The adjacency matrix for the second level is represented in Table 2 and its graphical representation is shown in Figure 5.

The first six elements are source nodes because they are containers of information that contribute to the definition of other nodes, but they are not influenced by any other nodes in the current representation. Indeed, their information is derived from outside the matrix (standards, task analysis, design decisions or risk assessment).

Each node can be further broken down into third-level nodes in order to refine their knowledge content.

For example, the 'workspaces' (W) node (node number 7 in the set of second-level nodes) can be broken down as follow:

- Human space (HS): space where operators can perform their tasks during the operations.

- Robot space (RS): space where the robot system (including the end effector and workpiece) can perform its tasks during the operations.

- $\quad$ Load/Unload space (L/U): space required to load and unload supply material.

- Collaborative space (CS): space obtained from the intersection of the HS and RS spaces; within this space, contacts between robot and operator are allowed.

- Operational space (OS): space required to perform the whole task; it is the combination of the human and robot space and contains the collaborative space.

The connections between the third-level nodes are highlighted in Table 3, and the graphical representation is shown in Figure 6. It is easy to see that some are source nodes, whereas others are sink nodes. Indeed, in this representation, the starting point is the load/unload space; the operational and collaborative workspaces are sink points and are the final steps in the decisionmaking process in this subgraph.

This process should be replicated for all the second-level nodes in order to obtain a complete matrix that highlights the connections between all the nodes.

The result is a matrix that can be filled according to the specific application and task.

This tool's great utility and versatility is due to its easy organisation and the possibility of using a set of existing or ad-hoc developed algorithms.

\section{CONCLUSIONS}

In this paper, the contents of the ISO international standards for HRC workplaces are illustrated and the basis for an anthropocentric design approach as key to fully enabling humanrobot interaction and the collaborative environment is highlighted.

Two main elements are tackled: (i) the introduction of two elementary spaces (human and robot spaces) and (ii) their dynamic variations in terms of their shape, size and position. Furthermore, the human is assumed to be the key to enabling a real collaborative relationship. The identification of dedicated spaces for humans and robots can help to manage their interaction and to set them inside the workplace, overcoming the limitations connected to a safety-based approach, which has been introduced by the standards. Therefore, the presented approach is oriented towards the layout design process thanks to the use of elementary spaces. The purpose is to enable a collaborative environment where humans and robots can work in a synergic way.

The proposed approach can guide the design phase of collaborative workplaces because it satisfies both human needs, 
through an ergonomic workplace design, and productive needs, because of the consideration given to humans and robots together.

Indeed, innovative workplaces need design approaches that move from a safety-based design to a collaboration-based design. In this new context, the human is considered to be an element that improves the productivity capacity instead of an intruder. In this scenario, it is necessary to manage the concepts introduced above together with the safety prescriptions contained in the standards. For this reason, a multi-level graph-based approach has been used in order to: (i) define the functional requirements linked to the design parameters and associated with HRC and (ii) manage the dense network of interdependencies to drive the design process. Starting with the contents of the standards, the main elements of a collaborative workplace were identified. A decomposition from the first level was then carried out. For the second-level nodes, a categorisation of the elements that make up the workplace and their relationships was proposed. Finally, an example of third-level decomposition presented the main areas that make up the layout.

Thus, human-robot collaboration can open interesting scenarios for the future of manufacturing, leading to a higher level of flexibility and customisation. As a result, in the near future, HRC will likely be widely used in private and public fields.

Future perspectives point to the validation of collaborative workspaces and the possibility of dynamically updating working areas by using tools and methods, such as machine-learning algorithms and digital twins.

\section{ACKNOWLEDGEMENT}

This study was developed with the economic support of MIUR (Italian Ministry of Universities and Research) under the remit of project ARS01_00861, 'Integrated collaborative systems for smart factory - ICOSAF'.

\section{REFERENCES}

[1] V. Villani, F. Pini, F. Leali, C. Secchi, Survey on human-robot collaboration in industrial settings: safety, intuitive interfaces and applications, Mechatronics 55 (2018) pp. 248-266. DOI: https://doi.org/10.1016/i.mechatronics.2018.02.009

[2] C. Labate, G. Di Gironimo, F. Renno, Plasma facing components: a conceptual design strategy for the first wall in FAST tokamak, Nuclear Fusion 55 (2015) 113013 DOI https://doi.org/10.1088/0029-5515/55/11/113013

[3] G. Di Gironimo, D. Carfora, G. Esposito, A. Lanzotti, D. Marzullo, M. Siuko, Concept design of the DEMO divertor cassette-to-vacuum vessel locking system adopting a systems engineering approach, Fusion Engineering and Design 94(1) (2015) pp. $72-81$.

DOI: https://doi.org/10.1016/i.fusengdes.2015.03.039

[4] G. Di Gironimo, A. Lanzotti, D. Marzullo, G. Esposito, D. Carfora, M. Siuko, Iterative and participative axiomatic design process in complex mechanical assemblies: case study on fusion engineering, International Journal on Interactive Design and Manufacturing 9 (2015) pp. 325-338.

DOI: https://doi.org/10.1007/s12008-015-0270-7

[5] S. Patalano, A. Lanzotti, D. M. Del Giudice, F. Vitolo, S. Gerbino, On the usability assessment of the graphical user interface related to a digital pattern software tool, International Journal on Interactive Design and Manufacturing 11(3) (2017) pp. 457-469.

DOI: https://doi.org/10.1007/s12008-015-0287-y

[6] P. Franciosa, A. Palit, F. Vitolo, D. Ceglarek, Rapid response diagnosis of multi-stage assembly process with compliant nonideal parts using self-evolving measurement system, Procidia
CIRP 60 (2017) pp. 38-43.

DOI: https://doi.org/10.1016/i.procir.2017.01.035

[7] Q. Tan, Y. Tong, S. Wu, D. Li, Anthropocentric approach for smart assembly: integration and collaboration, Journal of Robotics 2019 (2019), pp. 1-8.

DOI: https://doi.org/10.1155/2019/3146782

[8] I. Aaltonen, T. Salmi, I. Marstio, Refining levels of collaboration to support the design and evaluation of human-robot interaction in the manufacturing industry, Proc. of $51^{\text {st }}$ CIRP Conference on Manufacturing Systems, Stockholm, Sweden, 15-18 May 2018, pp. 93-98.

DOI: https://doi.org/10.1016/i.procir.2018.03.214

[9] J. Krüger, B. Nickolay, P. Heyer, G. Seliger, Image based 3D surveillance for flexible man-robot-cooperation, CIRP Annals 54 (2005) pp. 19-22.

DOI: https://doi.org/10.1016/S0007-8506(07)60040-7

[10] E. Helms, R. D. Schraft, M. Hagele, Robot assistant in industrial environments, Proc. of the $11^{\text {th }}$ IEEE International Workshop on Robot and Human Interactive Communication, Berlin, Germany, 2002, pp. 399-404. DOI: https://doi.org/10.1109/ROMAN.2002.1045655

[11] J. Krüger, T. K. Lien, A. Verl, Cooperation of human and machines in assembly lines, CIRP Annals - Manufacturing Technology 58 (2009) pp. 628-646. DOI: https://doi.org/10.1016/j.cirp.2009.09.009

[12] D. Bortot, M. Born, K. Bengler, Directly or on detours? How should industrial robots approximate humans?, Journal of Experimental Psychology 55 (2013) pp. 352-358. DOI: https://doi.org/10.1109/HRI.2013.6483515

[13] ISO, ISO/TS 15066: 2016: Robots and robotic devices Collaborative robots, Geneva, Switzerland: International Organization for Standardization, 2016.

[14] ISO, ISO 10218-1: 2011: Robots and robotic devices - Safety requirements for industrial robots - Part 1: Robots. Geneva, Switzerland: International Organization for Standardization, 2011.

[15] ISO, ISO 10218-2: 2011: Robots and robotic devices - Safety requirements for industrial robots - Part 2: Robot systems and integration. Geneva, Switzerland: International Organization for Standardization, 2011.

[16] L. Gualtieri, E. Rauch, R. Rojas, R. Vidoni, D. T. Matt, Application of axiomatic design for the design of a safe collaborative human-robot assembly workplace, MATEC Web of Conferences, 223, EDP Sciences, 2018, 01003, 8 pages. DOI: https://doi.org/10.1051/matecconf/201822301003

[17] J. O. Oyekan, W. Hutabarat, A. Tiwari, R. Grech, M. H. Aung, M. P. Mariani, L. López-Dávalos, T. Ricaud, S. Singh, C. Dupuis, The effectiveness of virtual environments in developing collaborative strategies between industrial robots and humans, Robotics and Computer-Integrated Manufacturing 55 (2019) pp. 41-54. DOI: https://doi.org/10.1016/i.rcim.2018.07.006

[18] T. Arai, R. Kato, M. Fujita, Assessment of operator stress induced by robot collaboration in assembly, Robotics and ComputerIntegrated Manufacturing 59 (2010) pp. 5-8. DOI: https://doi.org/10.1016/i.cirp.2010.03.043

[19] G. Michalos, S. Makris, P. Tsarouchi, T. Guasch, D. Kontovrakis, G. Chryssolouris, Design considerations for safe human-robot collaborative workplaces, Procedia CIRP 37 (2015) pp. 248-253. DOI: https://doi.org/10.1016/j.procir.2015.08.014

[20] M. Bdiwi, M. Pfeifer, A. Sterzing, A new strategy for ensuring human safety during various levels of interaction with industrial robots, CIRP Annals 66 (2017) pp. 453-456.

DOI: https://doi.org/10.1016/i.cirp.2017.04.009

[21] O. Ogorodnikova, Human weaknesses and strengths in collaboration with robots, Periodica Polytechnica, Mechanical Engineering 52 (2008) pp. 25-33. DOI: https://doi.org/10.3311/pp.me.2008-1.05

[22] A. Rega, S. Patalano, F. Vitolo, S. Gerbino, A sensor data fusionbased locating method for reverse engineering scanning systems, II Workshop on Metrology for Industry 4.0 and IoT (MetroInd4. 
0\&IoT), Naples, Italy, 2019, pp. 123-126.

DOI: https://doi.org/10.1109/METROI4.2019.8792864

[23] G. Michalos, S. Makris, J. Spiliotopoulos, I. Misios, P. Tsarouchi, G. Chryssolouris, ROBO-PARTNER: Seamless human-robot cooperation for intelligent, flexible and safe operations in the assembly factories of the future, Conference on Assembly Technologies and Systems, Procedia CIRP, Dresden, Germany, 13-14 November 2014, Volume 23, pp. 71-76.

DOI: https://doi.org/10.1016/i.procir.2014.10.079

[24] S. Patalano, F. Vitolo, A. Lanzotti, A digital pattern approach to 3D CAD modelling of automotive car door assembly by using directed graphs, Graph-Based Modelling in Engineering, Mechanisms and Machine Science 42 (2017) pp. 175-185. DOI: https://doi.org/10.1007/978-3-319-39020-8 13
[25] DNarsingh Deo, Graph theory with applications to engineering and computer science, Courier Dover Publications, Englewood Cliffs, New Jersey, 2017, ISBN 0486820815, 9780486820811, 496 pages.

[26] M. Koutrouli, E. Karatzas, D. Paez-Espino, G. A. Pavlopoulos, A guide to conquer the biological network era using graph theory, Frontiers in Bioengineering and Biotechnology 8 (2020) article 34. DOI: https://doi.org/10.3389/fbioe.2020.00034

[27] A. Majeed, I. Rauf, Graph theory: a comprehensive survey about graph theory applications in computer science and social networks, Inventions 5(1) (2020) article 10. DOI: https://doi.org/10.3390/inventions5010010

[28] S. E. Schaeffer, Graph clustering, Computer Science Review 1(1) (2007) pp. 27-64.

DOI: https://doi.org/10.1016/i.cosrev.2007.05.001 